\title{
The Canonical Variables, the Symplectic Structure and the Initial Value Formulation of the Generalized Einstein-Cartan Theory of Gravity
}

\author{
Wiktor Szczyrba \\ Institute of Mathematics, Polish Academy of Sciences, PL-00-950 Warsaw, Poland
}

\begin{abstract}
Canonical variables for the generalized (non-metric) Einstein-Cartan theory of gravity are defined. The space of solutions is equipped with a closed differential 2-form $\Omega$. The symplectic 2-form $\Omega$ has a diagonal representation in terms of canonical variables. A geometric interpretation of the canonical variables is presented and the $3+1$ formulation of the field equations is given.
\end{abstract}

\section{Introduction}

The canonical formulation of general relativity is based on the Palatini variational principle $[2,15,23]$. Also for simple examples of the gravitational field interacting with the scalar or electromagnetic field natural generalizations of the ADM formalism exist. In a more general situation the Lagrange function of the matter field does depend on coefficients of an affine connection. Therefore for such a theory the Palatini variational principle gives raise to a non-riemannian geometry. The most general case is when we admit a non-symmetric connection $\Gamma=\left(\Gamma_{\mu \nu}^{\lambda}\right)$ in space-time. Generalized theories of gravity were investigated by several authors: E. Cartan, Kibble, Sciama, Hehl, Trautman and others. We refer the reader to [7, 21] for a detailed bibliography. The present paper is based on the simplest generalization of the Palatini variational principle

$$
\delta \int \sqrt{-g}(R+\mathscr{L}) d^{4} x=0
$$

where a metric tensor $\boldsymbol{g}=\left(g_{\mu \nu}\right)$, a non-symmetric connection $\boldsymbol{\Gamma}=\left(\Gamma_{\mu \nu}^{\lambda}\right)$, a tensor $\boldsymbol{\varphi}=\left(\varphi^{A}\right)$ and its partial derivatives $\boldsymbol{\eta}=\left(\eta_{\mu}^{A}\right)$ are taken as independent variables. We formulate the variational principle (1.1) in the geometric language of differential forms and multisymplectic manifolds $[4,6,9-11,16-20]$. Our field equations derived from this principle coincide with those given by Trautman [21] cf. also [8].

It is known from the geometric theory of the calculus of variations and the theory of multisymplectic manifolds $[5,10,11]$ that the set of solutions of the field equations has a natural symplectic structure. This is the reason why we use such 
equations and do not impose the metric condition $D_{\lambda} g_{\mu \nu}=0 \mathrm{cf}$. [13, 22]. We call the theory obtained by the Palatini principle (1.1) the generalized Einstein-Cartan theory. It is known that in the classical general relativity the symplectic 2-form $\Omega$ can be diagonalized in the ADM coordinates $\left(\pi^{i j}, g_{i j}\right)$, where $\overline{\boldsymbol{g}}=\left(g_{i j}\right)$ is a metric tensor of 3-dimensional surface $\sigma$ in space-time $M$ and the ADM density $\pi=\left(\pi^{i j}\right)$ is defined by the second fundamental form $K=\left(K_{i j}\right)$ of the imbedding $i: \sigma \rightarrow M$ as follows

$$
\pi^{i j}=-\left(\operatorname{det} g_{i j}\right)^{1 / 2}\left(K_{a b} \bar{g}^{a i} \bar{g}^{b j}-\bar{g}^{i j} K_{a b} \bar{g}^{a b}\right) .
$$

More precisely we have

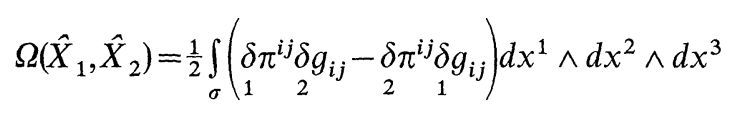

where $\underset{k}{\delta} \pi^{i j}, \underset{k}{\delta} g_{i j} k=1,2$ are "components" of tangent vectors $\hat{X}_{1}, \hat{X}_{2}$ cf. [17-19].

In the present paper we generalize the ADM density $\pi=\left(\pi^{i j}\right)$ for the case of interacting gravitational and tensor fields. The generalized ADM density $\Pi=\left(\Pi^{i j}\right)$ has a natural geometric interpretation in terms of the fundamental mappings of the hypersurface $\sigma \subset M$. We define also the canonical variables $\left(\overline{\mathscr{P}}_{A}, \bar{\varphi}^{A}\right)$ for the matter field $\varphi=\left(\varphi^{A}\right)$. In the canonical variables $\left(\Pi^{i j}, g_{i j}, \overline{\mathscr{P}}_{A}, \bar{\varphi}^{A}\right)$ the symplectic 2 -form $\Omega$ has a diagonal representation of the type (1.3).

It turns out that the generalized Einstein-Cartan equations

$$
G^{\mu \nu}-8 \pi \stackrel{s}{T}^{\mu \nu}=0
$$

splits into 6 dynamical equations and 4 constraints

$$
G_{\lambda}^{0}-8 \pi \stackrel{s}{T_{\lambda}^{0}}=0
$$

Equations (1.5) do not contain time derivatives and are constraints imposed on initial data of the canonical variables. This fact gives raise to degeneracy of the symplectic 2-form $\Omega$. Problems concerning the dynamical structure of the EinsteinCartan equations (cf. corresponding results for the Einstein theory in [3, 14]) and the degeneracy of the symplectic 2-form (cf. [17-19]) will be discussed elsewhere.

The methods we use in the present paper are similar to those used in $[10,17-$ 19]. Some technical results (the contracted Bianchi identities, the reduction of the system (1.4)) were proved in [20]. The notation follows that of $[9,10,12,15,17-$ 19] with the only exception, a different interpretation of the symbol $\frac{\partial}{\partial g_{\mu \nu}}$ (see
Appendix 1).

\section{The Field Equations}

Let $M$ be a 4 dimensional, smooth manifold-space-time, with local coordinates $\left(x^{\lambda}\right)$. Our geometric approach is based on the bundle $\tau_{1}: \mathscr{P}_{1} \rightarrow M$. A fibre of this bundle over a point $\boldsymbol{x} \in M$ is a direct sum of the space of symmetric 2 covariant tensors $\boldsymbol{g}=\left(g_{\mu \nu}\right)$ at $\boldsymbol{x}$ and the space of non-symmetric affine connections $\boldsymbol{\Gamma}=\left(\Gamma_{\mu \nu}^{\lambda}\right)$. 
The transformation properties of local coordinates $\left(x^{\lambda}, g_{\mu v}, \Gamma_{\mu \nu}^{\lambda}\right)$ in $\mathscr{P}_{1}$ are

$$
\begin{aligned}
g_{\mu^{\prime} v^{\prime}} & =\frac{\partial x^{\alpha}}{\partial x^{\mu^{\prime}}} \frac{\partial x^{\beta}}{\partial x^{\nu^{\prime}}} g_{\alpha \beta} \\
\Gamma_{\mu^{\prime} v^{\prime}}^{\lambda^{\prime}} & =\frac{\partial x^{\lambda^{\prime}}}{\partial x^{\tau}} \frac{\partial x^{\alpha}}{\partial x^{\mu^{\prime}}} \frac{\partial x^{\beta}}{\partial x^{v^{\prime}}} \Gamma_{\alpha \beta}^{\tau}+\frac{\partial^{2} x^{\sigma}}{\partial x^{\mu^{\prime}} \partial x^{v^{\prime}}} \frac{\partial x^{\lambda^{\prime}}}{\partial x^{\sigma}} .
\end{aligned}
$$

We have standard definitions of the Riemann tensor

$$
R_{\alpha \mu \nu}^{\beta}=\partial_{\mu} \Gamma_{v \alpha}^{\beta}-\partial_{v} \Gamma_{\mu \alpha}^{\beta}+\Gamma_{v \alpha}^{\tau} \Gamma_{\mu \tau}^{\beta}-\Gamma_{\mu \alpha}^{\tau} \Gamma_{v \tau}^{\beta}
$$

the Ricci tensor, the symmetrized Ricci tensor, the curvature scalar

$$
R_{\alpha \nu}=R_{\alpha \mu \nu}^{\mu} ; \quad{ }^{s} R_{\alpha \nu}=\frac{1}{2}\left(R_{\alpha \nu}+R_{\nu \alpha}\right) ; \quad R=g^{\mu \nu} R_{\mu \nu}
$$

the symmetric Einstein tensor

$$
G^{\mu \nu}=\frac{1}{2}\left(R^{\mu \nu}+R^{v \mu}\right)-\frac{1}{2} g^{\mu \nu} R
$$

the torsion tensor

$$
Q_{\mu \nu}^{\lambda}=\Gamma_{\mu \nu}^{\lambda}-\Gamma_{\nu \mu}^{\lambda} \text {. }
$$

A tensor field $\boldsymbol{g}=\left(g_{\mu \nu}\right)$ on $M$ defines the pseudo-riemannian connection $\gamma=\left(\gamma_{\mu \nu}^{\lambda}\right)$

$$
\gamma_{\mu \nu}^{\lambda}=\left\{\begin{array}{c}
\lambda \\
\mu \nu
\end{array}\right\}=\frac{1}{2} g^{\lambda \tau}\left(\partial_{\mu} g_{v \tau}+\partial_{\nu} g_{\mu \tau}-\partial_{\tau} g_{\mu \nu}\right) \text {. }
$$

Therefore we have two different covariant derivatives for tensor field on $M, D_{\mu}$ corresponding to the connection $\Gamma$ and $\nabla_{\mu}$ corresponding to $\gamma$.

The defect tensor is defined by

$$
r_{\mu \nu}^{\lambda}=\Gamma_{\mu \nu}^{\lambda}-\gamma_{\mu \nu}^{\lambda} \text {. }
$$

Very important in the subsequent considerations is the following tensor

$$
c_{\lambda}^{\mu \nu}=g^{\mu \nu} r_{\tau \lambda}^{\tau}+g^{\alpha \beta} r_{\alpha \beta}^{\nu} \delta_{\lambda}^{\mu}-g^{\tau \nu} r_{\tau \lambda}^{\mu}-g^{\tau \mu} r_{\lambda \tau}^{\nu} .
$$

It has the property $c_{\lambda}^{\mu \lambda}=0$, therefore $r_{\mu \nu}^{\lambda}$ can be expressed by $c_{\lambda}^{\mu \nu}$ only up to an arbitrary covector $\chi=\left(\chi_{\mu}\right)$

$$
\begin{aligned}
r_{\mu \nu}^{\lambda}= & -\chi_{\mu} \delta_{\nu}^{\lambda}-\frac{1}{4} g_{\alpha \beta} c_{\tau}^{\alpha \beta} g^{\tau \lambda} g_{\mu \nu}+\frac{1}{4} g_{\alpha \beta} c_{\nu}^{\alpha \beta} \delta_{\mu}^{\lambda} \\
& +-\frac{1}{4} c_{\tau}^{\tau \sigma} g_{\sigma \nu} \delta_{\mu}^{\lambda}+\frac{1}{4} c_{\tau}^{\tau \lambda} g_{\mu \nu}+\frac{1}{2} c_{\tau}^{\alpha \beta} g^{\tau \lambda} g_{\alpha \mu} g_{\beta \nu} \\
& +-\frac{1}{2} c_{\mu}^{\tau \lambda} g_{\tau \nu}-\frac{1}{2} c_{\nu}^{\lambda \tau} g_{\tau \mu} .
\end{aligned}
$$

In the following we consider the subbundle of $\mathscr{P}_{1}$ consisting of such systems $\left(x^{\lambda}, g_{\mu \nu}, \Gamma_{\mu \nu}^{\lambda}\right)$ that $g=\operatorname{det} g_{\mu \nu}<0$. We denote it also by $\mathscr{P}_{1}$. We have a differential 4 form $\stackrel{4}{\theta}_{g r}$ (the Cartan 4-form) on $\mathscr{P}_{1}$

$$
\begin{aligned}
\stackrel{4}{\theta}_{g r}= & (1 / 16 \pi)[g^{\alpha \beta} \sqrt{-g} d x^{0} \wedge \ldots \wedge \underbrace{d \Gamma_{\alpha \beta}^{\tau}}_{\tau} \wedge \ldots \wedge d x^{3} \\
& +-g^{\alpha \tau} \sqrt{-g} d x^{0} \wedge \ldots \wedge \underbrace{d \Gamma_{\beta \alpha}^{\beta}}_{\tau} \wedge \ldots \wedge d x^{3} \\
& \left.+g^{\mu \nu}\left(\Gamma_{v \mu}^{\tau} \Gamma_{\beta \tau}^{\beta}-\Gamma_{\beta \mu}^{\tau} \Gamma_{v \tau}^{\beta}\right) \sqrt{-g} d x^{0} \wedge \ldots \wedge d x^{3}\right] .
\end{aligned}
$$


By virtue of the transformation law (2.1) formula (2.10) defines globally a differential 4-form on $\mathscr{P}_{1}$. This $\stackrel{4}{\theta}_{g r}$ was given for the first time in $[17,18]$ for the case of symmetric connections.

Remark. If $f: M \rightarrow \mathscr{P}_{1}$ is a section of $\tau_{1}$ then $f^{*} \stackrel{4}{\theta}_{g r}=R \sqrt{-g} d x^{0} \wedge \ldots \wedge d x^{3}$, therefore $\stackrel{4}{\theta}_{g r}$ is the appropriate tool for the Palatini formulation of the variational principle in general relativity [18].

We need also another bundle $\tau_{2}: \mathscr{P}_{2} \rightarrow M$ which corresponds to variables of an external field (matter field). Let $\varrho: W \rightarrow M$ be a tensor bundle with local coordinates $\left(x^{\lambda}, \varphi^{A}\right)$, where $\varphi^{A}=\varphi_{\beta_{1} \ldots \beta_{s}}^{\alpha_{1} \ldots \alpha_{k}}$ have tensor transformation properties.

Let $\tau_{2}: \mathscr{P}_{2} \rightarrow M$ be the bundle of first jets of local sections of $W$ over $M$. In $\mathscr{P}_{2}$ we have local coordinates $\left(x^{\lambda}, \varphi^{A}, \eta_{\mu}^{A}\right)$ where $\eta_{\mu}^{A}$ have transformation properties of partial derivatives of $\varphi^{A}$. We define the multisymplectic bundle $\mathscr{P}$ as the fibre product of $\mathscr{P}_{1}$ and $\mathscr{P}_{2}$ over $M, \mathscr{P}=\mathscr{P}_{1} \oplus \mathscr{P}_{2}, \tau: \mathscr{P} \rightarrow M$. The 4 -form $\stackrel{4}{\theta}_{\text {gr }}$ is defined on $\mathscr{P}$ by its pull-back from $\mathscr{P}_{1}$. The Cartan 4-form $\stackrel{4}{\theta}_{f}$ corresponding to the matter field $\varphi=\left(\varphi^{A}\right)$ is

$$
\begin{aligned}
\stackrel{4}{\theta}_{f}= & \sqrt{-g} \frac{\partial \mathscr{L}}{\partial \eta_{\mu}^{A}} d x^{0} \wedge \ldots \wedge \underbrace{d \varphi^{A}}_{\mu} \wedge \ldots \wedge d x^{3} \\
& +-\left(\frac{\partial \mathscr{L}}{\partial \eta_{\mu}^{A}} \eta_{\mu}^{A}-\mathscr{L}\right) \sqrt{-g} d x^{0} \wedge \ldots \wedge d x^{3}
\end{aligned}
$$

where $\mathscr{L}: \mathscr{P} \rightarrow \mathbb{R}$ is the Lagrange function of the matter field cf. $[4,6,9,11,16,18]$. Remark. Taking as a principle the independence of the interaction of points of space-time we assume that in any coordinate system $\frac{\partial \mathscr{L}}{\partial x^{\lambda}}=0$.

The Cartan 4-form $\stackrel{4}{\theta}$ for the interacting gravitational and matter fields is

$$
\stackrel{4}{\theta}=\stackrel{4}{\theta}_{g r}+\stackrel{4}{\theta}_{f}
$$

The variational principle based on $\stackrel{4}{\theta}$ gives raise to the following definition:

The states of the system are sections $f: M \rightarrow \mathscr{P}$ satisfying the condition that for every $\tau$-vertical vector field $X$ tangent to $\mathscr{P}$ at points of the submanifold $C=f(M) \subset \mathscr{P}$

$$
\left.f^{*}(X\lrcorner d \stackrel{4}{\theta}\right)=0
$$

where $f^{*}$ is the cotangent mapping to $f \mathrm{cf}$ [4, 6, 9-11, 16-19].

In local coordinates Equation (2.13) reads

$$
\begin{aligned}
c_{\lambda}^{\mu \nu} & =-16 \pi s_{\lambda}^{\mu \nu}, \\
G^{\mu \nu} & =8 \pi \stackrel{s}{T}^{\mu \nu}, \\
\eta_{\mu}^{A} & =\partial_{\mu} \varphi^{A}, \\
(E-L)_{A} & =\frac{\partial \mathscr{L}}{\partial \varphi^{A}}-(-g)^{-1 / 2} \partial_{\mu}\left(\sqrt{-g} \frac{\partial \mathscr{L}}{\partial \eta_{\mu}^{A}}\right)=0,
\end{aligned}
$$


where

$$
s_{\lambda}^{\mu \nu}=\frac{\partial \mathscr{L}}{\partial \Gamma_{\mu \nu}^{\lambda}}
$$

is the hypermomentum tensor,

$$
\stackrel{s}{T}^{\mu v}=2 \frac{\partial \mathscr{L}}{\partial g_{\mu \nu}}+g^{\mu v} \mathscr{L}
$$

is the symmetric stress-energy tensor.

The system (2.14), (2.15) is identical with that obtained in $[13,21]$ by means of the classical Palatini variational principle, cf. also [8]. We call $(2.14),(2.15)$ the generalized Einstein-Cartan equations.

Equations (2.14a), (2.15a) are algebraic and allow us to express uniquely (or almost uniquely) $\Gamma_{\mu \nu}^{\lambda}$ by $g_{\mu \nu}, \partial_{\lambda} g_{\mu \nu}, \varphi^{A}, \partial_{\mu} \varphi^{A} \mathrm{cf}$. [20] where examples are given. In general the connection we get from these equation is non-metric i.e. $D_{\lambda} g_{\mu \nu} \neq 0$.

Remark. The physical interpretation of the tensors $s_{\lambda}^{\mu \nu}, \stackrel{s}{T}^{\mu \nu}$ was discussed in several papers $[7,8,21]$. We do not consider this problem here.

Now we impose some restrictions on the Lagrange function $\mathscr{L}$. It is natural to assume that $\mathscr{L}$ can be expressed by metric $g$ field $\varphi$, its covariant derivatives and torsion i.e.

$$
\mathscr{L}\left(g_{\mu \nu}, \Gamma_{\mu \nu}^{\lambda}, \varphi^{A}, \eta_{\mu}^{A}\right)=\mathscr{L}_{1}\left(g_{\mu \nu}, \varphi^{A}, D_{\lambda} \varphi^{A}, Q_{\mu \nu}^{\lambda}\right)
$$

For a tensor field $\varphi^{A}=\varphi_{\beta_{1} \ldots \beta_{s}}^{\alpha_{1} \ldots \alpha_{k}}$ we have from (2.18)

$$
\begin{aligned}
\frac{\partial \mathscr{L}}{\partial \Gamma_{\mu \nu}^{\lambda}}= & p_{\lambda \alpha_{2} \ldots \alpha_{k}}^{\mu \beta_{1} \ldots \beta_{s}} \varphi_{\beta_{1} \ldots \beta_{s}}^{v \alpha_{2} \ldots \alpha_{k}}+\ldots+p_{\alpha_{1} \ldots \alpha_{k} \lambda}^{\mu \beta_{1} \ldots \beta_{s}} \varphi_{\beta_{1} \ldots \beta_{s}}^{\alpha_{1} \ldots \alpha_{k}-1 v} \\
& +-p_{\alpha_{1} \ldots \alpha_{k}}^{\mu \nu \beta_{2} \ldots \beta_{s}} \varphi_{\lambda \beta_{2} \ldots \beta_{s}}^{\alpha_{1} \ldots \alpha_{k}}-\ldots-p_{\alpha_{1} \ldots \alpha_{k}}^{\mu \beta_{1} \ldots \beta_{s-1} v} \varphi_{\beta_{1} \ldots \beta_{s-1} \lambda}^{\alpha_{1} \ldots \alpha_{k}}+a_{\lambda}^{\mu v},
\end{aligned}
$$

where

$$
p_{\alpha_{1} \ldots \alpha_{k}}^{\mu \beta_{1} \ldots \beta_{s}}=\frac{\partial \mathscr{L}}{\partial \eta_{\mu \beta_{1} \ldots \beta_{s}}^{\alpha_{1} \ldots \alpha_{k}}}
$$

is the 4-momentum of the field $\varphi$ and $a_{\lambda}^{\mu \nu}$ is a tensor skew-symmetric in upper indices i.e. $a_{\lambda}^{v \mu}=-a_{\lambda}^{\mu \nu}$.

\section{The Contracted Bianchi Identities and the Reduced System of the Einstein-Cartan Equations}

The group of diffeomorphisms of space-time acts in a natural way in the bundle $\mathscr{P}$

$$
(\operatorname{Diff} M, \mathscr{P}) \ni(\Phi, p) \rightarrow A(\Phi)(p) \in \mathscr{P} \text {. }
$$

We assume that the theory is invariant with respect to this action. It means

$$
(A(\Phi))^{*} \stackrel{4}{\theta}=\stackrel{4}{\theta}
$$


It is easy to see that the definition of $\stackrel{4}{\theta}_{g r}$ implies

$$
(A(\Phi)) * \stackrel{4}{\theta}_{g r}=\stackrel{4}{\theta}_{g r}
$$

Therefore we get

$$
(A(\Phi)) * \stackrel{4}{\theta}_{f}=\stackrel{4}{\theta}_{f}
$$

The postulate (3.4) gives raise to several relations among metric, connection: stress-energy tensor, hypermomentum tensor and their derivatives. These relations known as the Belinfante-Rosenfeld identities were given for the Einstein-Cartan theory in $[20,21]$. It was proved in [20] that they give the following result (cf. also [21]).

Proposition 1. If the kinematical Equation (2.14a) and the Euler-Lagrange Equations (2.15a) and (2.15b) are satisfied then for every metric $g=\left(g_{\mu \nu}\right)$ and every connection $\Gamma=\left(\Gamma_{\mu \nu}^{\lambda}\right)$ on $M$ we have

$$
\nabla_{\tau}\left(G_{\lambda}^{\tau}-8 \pi \stackrel{s}{T_{\lambda}^{\tau}}\right)=0
$$

For our theory it is necessary to assume that $M=\mathbb{R} \times \sigma$ where $\sigma$ is a 3-dimensional manifold. We shall consider only such metrics on $M$ that $\sigma$ is a space-like submanifold and coordinate systems such that $\sigma=\left\{\boldsymbol{x} \in M: x^{0}=0\right\}$. By similar methods as those used in the classical general relativity $[1,18,19]$ it is easy to prove from Proposition 1.

Proposition 2 [20]. The Equations (2.14), (2.15) are equivalent to the system

$$
\begin{aligned}
& \eta_{\mu}^{A}=\partial_{\mu} \varphi^{A}, \quad(E-L)_{A}=0 \\
& c_{\lambda}^{\mu \nu}=-16 \pi s_{\lambda}^{\mu \nu} \\
& { }^{s} R_{i j}-8 \pi\left(\stackrel{s}{T}_{i j}-\frac{1}{2} g_{i j}\left(\stackrel{s}{T}^{\alpha \beta} g_{\alpha \beta}\right)\right)=0, \\
& G_{\lambda}^{0}-8 \pi \stackrel{s}{T}_{\lambda}^{0}=0 \quad \text { on } \sigma .
\end{aligned}
$$

We shall prove in Section 6 that Equations (3.7) do not contain $x^{0}$-derivatives and are constraints for initial data. If they are satisfied for $x^{0}=0$ they hold for all $x^{0}$.

\section{The Orthogonal Decomposition of Tensors Associated with a Given Space-like Surface in $M$}

Let $\boldsymbol{g}=\left(g_{\mu \nu}\right)$ be a pseudoriemannian metric on $M$ with the signature $(-1,+3)$, $\sigma=\left\{\boldsymbol{x} \in M: x^{0}=0\right\}$ be a 3-dimensional space-like surface in $M$ and $\boldsymbol{n}=\left(n^{\mu}\right)$ be the normal unit vector to $\sigma$ at $\boldsymbol{x}=\left(x^{\lambda}\right)(\boldsymbol{n} \cdot \boldsymbol{n}=-1)$.

Let $\varphi=\left(\varphi_{\beta_{1} \ldots \beta_{s}}^{\alpha_{1} \ldots \alpha_{k}}\right)$ be a tensor (tangent/cotangent to $\left.M\right)$ at $x \in \sigma$. The idea of the orthogonal decomposition of $\varphi$ is based on the notion of the gaussian (normal) coordinate system. Let $\left(x^{\bar{\lambda}}\right)$ be a coordinate system in a neighbourhood of $\sigma$ in $M$ such that $\sigma=\left\{\boldsymbol{x} \in M: x^{\overline{0}}=0\right\}$ and $\boldsymbol{n}=(1,0,0,0)$ on $\sigma$. Coordinates $\varphi_{\bar{\beta}_{1} \ldots \bar{\beta}_{s}}^{\bar{\alpha}_{1} \ldots \bar{\alpha}_{k}}$ of $\boldsymbol{\varphi}$ in this system give components of several tensors tangent/cotangent to $\sigma$. 
Their valence is determined by the number of non-zero indices in $\varphi_{\bar{\beta}_{1} \ldots \bar{\beta}_{s}}^{\bar{\alpha}_{1} \ldots \bar{x}_{k}}$ According to the classical terminology $[2,23]$ we call geometric objects tangent/ cotangent to $M$ the 4 objects and geometric objects tangent/cotangent to $\sigma$ the 3objects. The 3-object generated by a 4-object $\varphi=\left(\varphi_{\beta_{1} \ldots \beta_{s}}^{\alpha_{1} \ldots \alpha_{k}}\right)$ is denoted by $\bar{\varphi}=\left(\bar{\varphi}_{\beta_{1} \ldots \beta_{s}}^{\alpha_{1} \ldots \alpha_{k}}\right)$ where

$$
\bar{\varphi}_{\beta_{1} \ldots \beta_{s}}^{\alpha_{1} \ldots \alpha_{k}}=\varphi_{\bar{\beta}_{1} \ldots \bar{\beta}_{s}}^{\bar{\alpha}_{1} \ldots \bar{\alpha}_{k}}=\frac{\partial x^{\bar{\alpha}_{1}}}{\partial x^{\mu_{1}}} \ldots \frac{\partial x^{\bar{\alpha}_{k}}}{\partial x^{\mu_{k}}} \cdot \frac{\partial x^{v_{1}}}{\partial x^{\beta_{1}}} \ldots \frac{\partial x^{v_{s}}}{\partial x^{\bar{\beta}_{s}}} \varphi_{v_{1} \ldots v_{s}}^{\mu_{1} \ldots \mu_{k}} .
$$

Remarks. The matrices $\left[\frac{\partial x^{\bar{\alpha}}}{\partial x^{\mu}}\right],\left[\frac{\partial x^{\nu}}{\partial x^{\bar{\beta}}}\right]$ are given in Appendix 2.

Examples. (i) for a vector $\boldsymbol{v}=\left(v^{\alpha}\right)$ we have the scalar $\bar{v}^{0}=v^{\overline{0}}=-\boldsymbol{n} \cdot \boldsymbol{v}$ (the projection $\boldsymbol{v}$ on $\boldsymbol{n}$ ) the vector (tangent to $\sigma$ ) $\bar{v}^{k}=v^{\bar{k}}=v^{k}+(\boldsymbol{v} \cdot \boldsymbol{n}) n^{k}$ (the projection of $\boldsymbol{v}$ on $\sigma$ ).

(ii) For a covector $\boldsymbol{p}=\left(p_{\alpha}\right)$ we have the scalar $\bar{p}_{0}=\langle\boldsymbol{n} \mid \boldsymbol{p}\rangle=n^{\alpha} p_{\alpha}$ the covector $\bar{p}_{k}=p_{k}$ (the pull-back of $\boldsymbol{p}$ on $\sigma$ ).

(iii) For the metric $\boldsymbol{g}=\left(g_{\mu v}\right)$

$$
\begin{array}{lll}
\bar{g}_{00}=-1, & \bar{g}_{0 k}=\bar{g}_{k 0}=0, & \bar{g}_{p q}=g_{p q}, \\
\bar{g}^{00}=-1, & \bar{g}^{0 k}=\bar{g}^{k 0}=0, & \bar{g}^{p q}=\left(g^{-1}\right)^{p q},
\end{array}
$$

where $\left[\left(g^{-1}\right)^{p q}\right]$ is the inverse matrix of $\left[g_{p q}\right]$.

We define also

$$
\bar{g}=\operatorname{det} g_{p q} \text {. }
$$

The orthogonal decompositions are compatible with the natural pairings of vectors and covectors e.g.

$$
\langle\boldsymbol{v} \mid \boldsymbol{p}\rangle=v^{\alpha} p_{\alpha}=\bar{v}^{0} \bar{p}_{0}+\bar{v}^{k} \bar{p}_{k} .
$$

In Appendix 2 we present several relations between 3- and 4-objects.

\section{Canonical Variables for the Interacting Gravitational and Tensor Fields}

For a pseudoriemannian metric $\boldsymbol{g}=\left(g_{\mu \nu}\right)$ on $M$ and a space-like surface $\sigma=\left\{\boldsymbol{x} \in M: x^{0}=0\right\}$ we define the lapse function $N=\left(-g^{00}\right)^{-1 / 2}$, the shift covector field $N_{k}=g_{0 k}$ and the metric tensor $\overline{\boldsymbol{g}}=\left(g_{i j}\right)$. The components of the unit normal vector to $\sigma$ are $\left(n^{\mu}\right)=\left(1 / N,-N^{k} / N\right)$. A change of $\boldsymbol{n}$ caused by its parallel transport with respect to the riemannian connection $\gamma_{\mu \nu}^{\lambda}=\left\{\begin{array}{l}{ }_{\mu \nu} \\ \mu v\end{array}\right\}$ in a direction tangent to $\sigma$ defines the symmetric 2 covariant tensor field on $\sigma$ (the second fundamental form of $\sigma$ in the riemannian geometry)

$$
K_{i j}=-g_{j \mu} \nabla_{i} n^{\mu} .
$$

We know, cf. $[2,17-19,23]$ that for the pure gravitational field the canonical variables conjugate to the components of the metric $g_{i j}$ on $\sigma$ are given by the ADM density on $\sigma$

$$
\pi^{i j}=-\sqrt{\bar{g}}\left(K_{p q}-g_{p q} K_{a b} \bar{g}^{a b}\right) \bar{g}^{p i} \bar{g}^{q j} .
$$


It was proved in $[17,18]$ that the symplectic form in the set of solution of Einstein equations is, in terms of the variables $\left(\pi^{i j}, g_{i j}\right)$ diagonal. The corresponding discussion for the Einstein-Cartan theory gives raise to the following definition of the canonical momenta (cf. Section 7)

$$
\begin{aligned}
\Pi^{p q}= & \pi^{p q}+\frac{1}{4} \sqrt{-g}\left(c_{\tau}^{p q}+c_{\tau}^{q p}\right) g^{\tau 0}-\frac{1}{4} \sqrt{-g}\left(c_{\tau}^{p 0}+c_{\tau}^{0 p}\right) g^{\tau q} \\
& +-\frac{1}{4} \sqrt{-g}\left(c_{\tau}^{q 0}+c_{\tau}^{0 q}\right) g^{\tau p}-\frac{1}{2} \sqrt{-g}\left(c_{\tau}^{00} g^{\tau p} N^{q}+c_{\tau}^{00} g^{\tau q} N^{p}\right) \\
& +-\frac{1}{2} \sqrt{-g} c_{\tau}^{00} g^{\tau 0} N^{p} N^{q} .
\end{aligned}
$$

If

$$
z_{\lambda}^{\mu \nu}=c_{\lambda}^{\mu \nu}+16 \pi a_{\lambda}^{\mu \nu}
$$

then by virtue of the skew symmetry properties of $a_{\lambda}^{\mu \nu}$ we have (cf. Appendix 2)

$$
\begin{aligned}
\Pi^{p q}= & \pi^{p q}-\frac{1}{4} \sqrt{\bar{g}}\left(\bar{z}_{0}^{p q}+\bar{z}_{0}^{q p}\right) \\
& +-\frac{1}{4} \sqrt{\bar{g}}\left(\left(\bar{z}_{\boldsymbol{r}}^{p 0}+\bar{z}_{\boldsymbol{r}}^{0 p}\right) \bar{g}^{r q}+\left(\bar{z}_{\boldsymbol{r}}^{q 0}+\bar{z}_{\boldsymbol{r}}^{0 q}\right) \bar{g}^{r p}\right) .
\end{aligned}
$$

For the matter field $\varphi$ we take as the canonical variables

$$
\bar{\varphi}_{\beta_{1} \ldots \beta_{s}}^{\alpha_{1} \ldots \alpha_{k}}, \quad \overline{\mathscr{P}}_{\alpha_{1} \ldots \alpha_{k}}^{\beta_{1} \ldots \beta_{s}}=\sqrt{\bar{g}} \bar{p}_{\alpha_{1} \ldots \alpha_{s}}^{0 \beta_{1} \ldots \beta_{s}} .
$$

Remark. It follows from (2.19), (2.20), (2.16), and (2.14a) that $z_{\lambda}^{\mu \nu}$ can by expressed by $p_{A}^{\lambda}, \varphi^{A}$, and $\sqrt{\bar{g}} \bar{z}_{\lambda}^{0 v}$ can be expressed by the canonical variables $\left(\overline{\mathscr{P}}_{A}, \bar{\varphi}^{A}\right)$.

\section{The Field Equations of the Gravitational Field in the Canonical Variables}

Proposition 3. If the kinematical Equation (2.14a) and the Euler-Lagrange Equations (2.15a) and (2.15b) hold then the constraints (3.7) read

$$
\begin{aligned}
& N \sqrt{\bar{g}}\left(G_{r}^{0}-8 \pi \stackrel{s}{T}_{r}^{0}\right) \\
& =-\bar{\nabla}_{p} \Pi_{r}^{p}-\frac{1}{2} \bar{\nabla}_{p}\left(\sqrt{\bar{g}} \bar{z}_{r}^{0 p}\right)+8 \pi \overline{\mathscr{P}}_{\mu_{1} \ldots \mu_{k}}^{\nu_{1} \ldots v_{s}} \bar{\nabla}_{r} \bar{\varphi}_{v_{1} \ldots v_{s}}^{\mu_{1} \ldots \mu_{k}}=0, \\
& {\left[\left(G_{0}^{0}-8 \pi{\stackrel{s}{T_{0}^{0}}}^{0}\right)-N^{r}\left(G_{r}^{0}-8 \pi \stackrel{T}{T}_{r}^{0}\right)\right]} \\
& =-\frac{1}{2}{ }^{(3)} R+(1 / 2 \bar{g})\left[\Pi^{p q} \Pi_{p q}-\frac{1}{2}(\operatorname{tr} \Pi)^{2}\right] \\
& \quad+-(1 / 2 \bar{g})\left[\left(\Pi^{p q}-\pi^{p q}\right)\left(\Pi_{p q}-\pi_{p q}\right)-\frac{1}{2}(\operatorname{tr}(\Pi-\pi))^{2}\right] \\
& \quad+-(1 / 2 \sqrt{\bar{g}}) \bar{\nabla}_{p}\left(\sqrt{\bar{g}} \bar{z}_{0}^{0 p}\right)-(1 / 2 \sqrt{\bar{g}}) \bar{\nabla}_{p}\left(\sqrt{\bar{g}} \bar{z}_{s}^{00} \bar{g}^{s p}\right) \\
& \quad+(1 / 2 \bar{g})\left(\pi \tau_{p q}-\frac{1}{2} g_{p q} \operatorname{tr} \pi\right)\left(\sqrt{\bar{g}} \bar{z}_{s}^{0 p} \bar{g}^{s q}\right) \\
& \quad+-8 \pi \mathscr{L}+(8 \pi / \sqrt{\bar{g}}) \overline{\mathscr{P}}_{\mu_{1} \ldots \mu_{k}}^{v_{1} \ldots v_{s}} \bar{D}_{0} \varphi_{v_{1} \ldots v_{s}}^{\mu_{1} \ldots \mu_{k}} \\
& \quad+(1 / 2 \sqrt{\bar{g}})\left(\sqrt{\bar{g}} \bar{z}_{\tau}^{0 \lambda}\right) \bar{r}_{0 \lambda}^{\tau}-\frac{1}{4} \bar{c}_{\lambda}^{\alpha \beta} \bar{r}_{\alpha \beta}^{\lambda}=0 .
\end{aligned}
$$

Remark. In (6.1) and (6.2) ${ }^{(3)} R_{i j},{ }^{(3)} R$ denote the Ricci tensor and the curvature scalar of the riemannian geometry given by the metric tensor $\bar{g}=\left(g_{i j}\right)$ on $\sigma$. 
The 3-object $\bar{D}_{0} \varphi_{\beta_{1} \ldots \beta_{s}}^{\alpha_{1} \ldots \alpha_{k}}$ is defined by the general procedure given in Section 4 (cf. Appendix 2). $\bar{V}_{k}$ denotes the covariant derivative corresponding to the riemannian metric $\left(g_{i j}\right)$ on $\sigma$.

To get formulas (6.1a) and (6.1b) we have to use the Belinfante-Rosenfeld identities proved in $[20,21]$.

It is easy to see that Equations (6.1a) contain only canonical variables $\Pi^{p q}, g_{p q}$, $\overline{\mathscr{P}}_{A}, \bar{\varphi}^{A}$ and their $x^{k}$-derivatives. The same is true for Equation $(6.1 \mathrm{~b})$ but the proof of this fact is more complicated. One can prove that the linearized version of $(6.1 \mathrm{~b})$ (the "infinitesimal change") depends only on $\delta \Pi^{p q}, \delta g_{p q}, \delta \overline{\mathscr{P}}_{A}, \delta \bar{\varphi}^{A}$ and their $x^{k}$-derivatives. Therefore $(6.1 \mathrm{~b})$ depends only on canonical variables and their spatial derivatives.

The dynamical Equations (3.6) give raise to

Proposition 4. If Equations (2.14a) and the Euler-Lagrange Equations (2.15a) and (2.15b) hold then

$$
\begin{aligned}
\sqrt{-} & \left({ }^{s} R_{p q}-8 \pi\left(\stackrel{T}{p q}_{p q}-\frac{1}{2} g_{p q} \operatorname{tr} \stackrel{s}{T}\right)\right)^{p i} \bar{g}^{q j} \\
& +-\sqrt{-g} \bar{g}^{i j(s} R_{a b}-8 \pi\left(T_{a b}^{s}-\frac{1}{2} g_{a b} \operatorname{tr}{\stackrel{s}{T})) \bar{g}^{a b}}^{s}\right. \\
= & \partial_{0} \Pi^{i j}+N \sqrt{\bar{g}}\left({ }^{(3)} R^{i j}-\frac{1}{2} \bar{g}^{i j(3)} R\right) \\
& +-(N / 2 \sqrt{\bar{g}}) \bar{g}^{i j}\left(\pi_{a b} \pi^{a b}-\frac{1}{2}(\operatorname{tr} \pi)^{2}\right) \\
& +(2 N / \sqrt{\bar{g}})\left(\pi_{q}^{i} \pi^{q j}-\frac{1}{2} \pi^{i j} \operatorname{tr} \pi\right) \\
& +\bar{\nabla}_{p} N^{j} \Pi^{p i}+\bar{\nabla}_{p} N^{i} \Pi^{p j}-\bar{\nabla}_{p}\left(\Pi^{i j} N^{p}\right) \\
& +-\sqrt{\bar{g}}\left(\bar{\nabla}^{i} \bar{\nabla}^{j} N-\bar{g}^{i j} \bar{\nabla}^{k} \bar{\nabla}_{k} N\right) \\
& +\frac{1}{4} \sqrt{\bar{g}} \bar{\nabla}\left[N\left(\bar{z}_{q}^{i j}+\bar{z}_{q}^{j i}\right)\right] \bar{g}^{p q} \\
& +-\frac{1}{4} \sqrt{\bar{g}} \overline{V_{s}}\left[N\left(\bar{z}_{q}^{s i} \bar{g}^{q j}+\bar{z}_{q}^{i s} \bar{g}^{q j}+\bar{z}_{q}^{s j} \bar{g}^{q i}+\bar{z}_{q}^{j s} \bar{g}^{q i}\right)\right] \\
& +-\frac{1}{2} \sqrt{\bar{g}} \bar{z}_{q}^{00}\left(\bar{g}^{i a} \bar{g}^{q j}+\bar{g}^{j a} \bar{g}^{q i}\right) \partial_{a} N \\
& +-\frac{1}{2} N\left(\bar{z}_{q}^{0 p}+\bar{z}_{q}^{p 0}\right) \bar{g}^{q j} \bar{g}^{i u}\left(\pi_{u p}-\frac{1}{2} g_{u p} \operatorname{tr} \pi\right) \\
& +-\frac{1}{2} N\left(\bar{z}_{q}^{0 p}+\bar{z}_{q}^{p 0}\right) \bar{g}^{q i} \bar{g}^{j u}\left(\pi_{u p}-\frac{1}{2} g_{u p} \operatorname{tr} \pi\right) \\
& +\frac{1}{4} N \sqrt{\bar{g}} g_{q s}\left(-\bar{z}_{p}^{\alpha \beta} \bar{r}_{\alpha \beta}^{s}+\bar{z}_{\tau}^{\alpha s} \bar{r}_{\alpha p}^{\tau}+\bar{z}_{\tau}^{s \alpha} \bar{r}_{p \alpha}^{\tau}\right)\left(\bar{g}^{p i} \bar{g}^{q j}+\bar{g}^{p j} \bar{g}^{q i}\right) \\
& +N \sqrt{\bar{g}} g_{q s}\left(2 \pi \bar{a}_{p}^{\alpha \beta} \bar{Q}_{\alpha \beta}^{s}-4 \pi \bar{a}_{\tau}^{\alpha s} \bar{Q}_{\alpha p}^{\tau}\right)\left(\bar{g}^{p i} \bar{g}^{q j}+\bar{g}^{p j} \bar{g}^{q i}\right) \\
& +-\frac{1}{4} N \sqrt{\bar{g}} \bar{g}^{i j} \bar{c}_{\lambda}^{\alpha \beta} \bar{r}_{\alpha \beta}^{\lambda}-8 \pi N \sqrt{\bar{g}} \stackrel{T}{p q}_{p q} \bar{g}^{p i} \bar{g}^{q j}=0 .
\end{aligned}
$$

Equations (6.1), (6.2) together with (2.14a), (2.15a) and (2.15b) and

$$
\partial_{0} g_{i j}=\bar{\nabla}_{i} N_{j}+\bar{\nabla}_{j} N_{i}+\frac{2 N}{\sqrt{\tilde{g}}}\left(\pi_{i j}-\frac{1}{2} g_{i j} \operatorname{tr} \pi\right)
$$

give the complete system of field equations. We have no equations containing $\partial_{0} N, \partial_{0} N^{k}$ and therefore these variables are to be given in an arbitrary way on $M$. It is possible because the constraints (6.1) do not contain $N, N^{k}$. 


\section{The Symplectic Differential 2-Form on the Space of Solutions of the Generalized Einstein-Cartan Equations}

In our approach solutions of the field equations are sections of the multisymplectic (multiphase) bundle $\mathscr{P}$ satisfying (2.13). It is difficult to expect that Equation (2.13) has global solutions on $M$. Therefore we consider only sections of $\mathscr{P}$ over a neighbourhood of 3-dimensional surface $\sigma$ in $M$. The images of these sections form the space of states $\mathscr{H}$ (a set of 4-dimensional submanifolds in $\mathscr{P}$ ) equipped with a natural pseudodifferential structure $[10,18]$.

A vector $\hat{X}$ tangent to $\mathscr{H}$ at $C \in \mathscr{H}$ is represented by a $\tau$-vertical vector field $X$ tangent to $\mathscr{P}$ and defined on $C \subset \mathscr{P}$, which satisfies the linearized version of (2.13) cf. $[5,10,11,17-19]$. In local coordinates

$$
X=\delta g_{\mu \nu} \frac{\partial}{\partial g_{\mu \nu}}+\delta \Gamma_{\mu \nu}^{\lambda} \frac{\partial}{\partial \Gamma_{\mu \nu}^{\lambda}}+\delta \varphi^{A} \frac{\partial}{\partial \varphi^{A}}+\delta \eta_{\mu}^{A} \frac{\partial}{\partial \eta_{\mu}^{A}},
$$

where components $\delta g_{\mu \nu}, \delta \Gamma_{\mu \nu}^{\lambda}, \delta \varphi^{A}, \delta \eta_{\mu}^{A}$ are geometric objects on the submanifold $C$ (or equivalently on $M$ ) which satisfy the linearized version of $(2.14),(2.15)$.

Remark. $\delta g_{\mu \nu}, \delta \Gamma_{\mu \nu}^{\lambda}, \delta \varphi^{A}$ are tensor fields on $C$ (on $M$ ).

We define, cf. [18] the differential 1-form $\Theta$ on $\mathscr{H}$

$$
\left.\langle\hat{X} \mid \Theta\rangle=\int_{c} X\right\lrcorner \stackrel{4}{\theta}, \quad \hat{X} \in T_{C}(\mathscr{H}),
$$

where $c$ is the 3-dimensional submanifold in $C=f(M)$ corresponding to the spacelike surface $\sigma$ in $M(c=f(\sigma))$ and $X$ is a $\tau$-vertical vector field representing $\hat{X}$. The definition of $\Theta$ depends on the choice of a space-like surface $\sigma \subset M$ but its exterior derivative $\Omega=d \Theta$ given by

$$
\left.\Omega\left(\hat{X}_{1}, \hat{X}_{2}\right)=\frac{1}{2}\left\langle\left(\hat{X}_{1} \wedge \hat{X}_{2}\right) \mid \Omega\right\rangle=\frac{1}{2} \int_{c}\left(X_{1} \wedge X_{2}\right)\right\lrcorner d \dot{4}
$$

is independent of the choice of $\sigma \subset M([5,10,11,17-19])$.

Therefore the differential 2 -form $\Omega$ is well defined geometric object determined by the internal structure of the space $\mathscr{H}$.

Remark. Note a difference in the factor $1 / 2$ between (7.2), (7.3) and the corresponding formulas in [10, 17-19].

Theorem 1. If $\hat{X}_{1}, \hat{X}_{2}$ are vectors tangent to $\mathscr{H}$ at $C$ and $X_{1}, X_{2}$ are representing them $\tau$-vertical vector fields on $C$ then

$$
\begin{aligned}
& \Omega\left(\hat{X}_{1}, \hat{X}_{2}\right)=(1 / 32 \pi) \int_{c}\left({ }_{1}^{\delta} \Pi_{2}^{p q} \delta g_{p q}-\delta_{2} \Pi_{1}^{p q} \delta g_{p q}\right) d x^{1} \wedge d x^{2} \wedge d x^{3} \\
& +\frac{1}{2} \int_{c}\left(\underset{1}{\delta} \overline{\mathscr{P}}_{\alpha_{1} \ldots \alpha_{k}}^{\beta_{1} \ldots \beta_{s}} \delta \bar{\varphi}_{\beta_{1} \ldots \beta_{s}}^{\alpha_{1} \ldots \alpha_{k}}-\delta_{2} \overline{\mathscr{P}}_{\alpha_{1} \ldots \alpha_{k}}^{\beta_{1} \ldots \beta_{s}} \delta \bar{\varphi}_{\beta_{1} \ldots \beta_{s}}^{\alpha_{1} \ldots \alpha_{k}}\right) d x^{1} \wedge d x^{2} \wedge d x^{3},
\end{aligned}
$$

where $\delta_{i} \Pi^{p q},{ }_{i} g_{p q},{ }_{i} \overline{\mathscr{P}}_{A},{ }_{i} \bar{\varphi}^{A}$ are expressed by components of vector fields $X_{i} i=1,2$ using the linearized version of (4.1), (5.3), (5.5).

Remark. We assume that in (7.2)-(7.4) the components of the vector fields $X_{i}$ vanish rapidly at the spatial infinity such that the corresponding integrals exist. 
Formula (7.4) shows that the tensor density $\Pi=\left(\Pi^{p q}\right)$ is a good generalization of the ADM tensor density $\pi=\left(\pi^{p q}\right)$ for the case of the gravitational field interacting with matter.

Remark. If we take as an example the non-linear electrodynamics then the canonical variables $\left(\overline{\mathscr{P}}_{A}, \bar{\varphi}^{A}\right)$ coincide with those given in $[15,19]$.

In the next section we give the definition of the $\boldsymbol{\Pi}$ density in terms of geometric objects associated with an imbedded surface $\sigma \subset M$ and generalize the ADM interpretation of the density $\pi$ in classical general relativity.

Proof of Theorem 1. If a submanifold $C \subset \mathscr{P}$ is the image of a section $f: M \rightarrow \mathscr{P}$ and $f$ satisfies the kinematical Equation (2.14a) then

$$
\stackrel{4}{\theta} \mid C=\stackrel{4}{\theta}+\underset{1}{\stackrel{4}{2}}+\stackrel{4}{\theta}+\stackrel{4}{\theta}
$$

where

$$
\begin{aligned}
\underset{1}{\theta}= & {\left[(-1 / 16 \pi) g_{p q} d \Pi^{p q}+\overline{\mathscr{P}}_{\alpha_{1} \ldots \alpha_{k}}^{\beta_{1} \ldots \beta_{s}} d \bar{\varphi}_{\beta_{1} \ldots \beta_{s}}^{\alpha_{1} \ldots \alpha_{k}}\right] \wedge d x^{1} \wedge d x^{2} \wedge d x^{3}, } \\
\stackrel{4}{\theta}= & (1 / 16 \pi)\left[\sqrt{\bar{g}}(1 / N) \bar{g}^{k u}\left(d\left(\partial_{u^{\prime}} N_{k}\right)-\bar{\gamma}_{k u}^{s} d N_{s}-N_{s} d \bar{\gamma}_{k u}^{s}\right)\right. \\
& +\sqrt{\bar{g}}\left(\partial_{k}(1 / N) \bar{g}^{k u} d N_{u}-\partial_{k}(1 / N) \bar{g}^{q u} \bar{g}^{k r} N_{u} d g_{q r}\right) \\
& \left.+-\sqrt{\bar{g}}\left((1 / N) \bar{\nabla}_{u} N_{k} \bar{g}^{k r} \bar{g}^{q u} d g_{r q}+\left(N^{k} / N\right) d \bar{\gamma}_{p k}^{p}\right)\right] \wedge d x^{1} \wedge d x^{2} \wedge d x^{3}, \\
\stackrel{4}{\theta}= & (1 / 32 \pi)\left[-d\left(\sqrt{\bar{g}} N^{3} c_{\tau}^{00} g^{\tau 0}\right)\right] \wedge d x^{1} \wedge d x^{2} \wedge d x^{3}, \\
\stackrel{4}{\theta}= & \text { all terms in } \stackrel{4}{\theta} \text { which contain } d x^{0} .
\end{aligned}
$$

The form ${ }_{3}^{4}$ is closed, integrals containing $\underset{4}{4}$ over submanifolds $x^{0}=$ const. vanish and therefore we have to compute the integrals

$$
I_{1}=\frac{1}{2} \int_{c}\left(X_{1} \wedge X_{2}\right)-d \stackrel{4}{\theta}, \quad I_{2}=\frac{1}{2} \int_{c}\left(X_{1} \wedge X_{2}\right)-d \underset{2}{4} .
$$

It was proved in [17-19] that the integral $I_{2}$ can be transformed into an integral of an exact 3-form on $c$ i.e. $I_{2}=\int_{c} d \mu$ and therefore vanishes by virtue of boundary conditions on $c$ (on $\sigma$ ). The integral $I_{1}$ gives raise exactly to (7.4).

We know that the canonical variables are not independent, they satisfy at least 4 constraints (6.1). These constraints give raise to the degeneracy of the symplectic 2 -form $\Omega$. It can be proved by methods similar to those used in [17-19] that the degeneracy of $\Omega$ caused by (6.1) is determined by the action of Diff $M$ in the space $\mathscr{H}$. This result will be published elsewhere.

In particular cases other constraints can be given by the Euler-Lagrange equations, for instance in electrodynamics [19]. They give raise to an additional degeneracy of $\Omega$. 


\section{Fundamental Mappings of a Hypersurface in Space-Time}

Let $M$ be endowed with a pseudoriemannian metric $\boldsymbol{g}=\left(g_{\mu \nu}\right)$ and a non-symmetric connection $\Gamma=\left(\Gamma_{\mu \nu}^{\lambda}\right)$. Let $i: \sigma \rightarrow M$ be an imbedded 3-dimensional submanifold, which is space-like with respect to $g$ i.e. the induced metric (the first fundamental form of the imbedding)

$$
\overline{\boldsymbol{g}}=i^{*} \boldsymbol{g}
$$

is positively definite (riemannian).

Let $\boldsymbol{n}=\left(n^{\mu}\right)$ be the field of unit vectors normal to $\sigma$ i.e.

$$
(\boldsymbol{n} \mid \boldsymbol{n})=\boldsymbol{g}(\boldsymbol{n}, \boldsymbol{n})=-1 \text {. }
$$

For a vector $\boldsymbol{X}$ tangent to $\sigma$ at $\boldsymbol{x}$ and a vector field $\boldsymbol{Y}$ tangent to $\sigma$ we define the normal and tangent components of the covariant derivative $\boldsymbol{D}=\left(D_{\lambda}\right)$

$$
\begin{aligned}
& \left(D_{X} n\right)_{n}=-g\left(D_{X} n, n\right), \\
& \left(D_{X} n\right)_{t}=D_{X} n-\left(D_{X} n\right)_{n} n, \\
& \left(D_{X} Y\right)_{n}=-g\left(D_{X} Y, n\right), \\
& \left(D_{X} Y\right)_{t}=D_{X} Y-\left(D_{X} Y\right)_{n} n .
\end{aligned}
$$

From the properties of covariant derivatives we have

Proposition 5. The mapping

$$
\begin{aligned}
& C^{\infty}(T(\sigma)) \times C^{\infty}(T(\sigma)) \ni(X, Y) \rightarrow S(X, Y) \\
& \quad=-\left(D_{\boldsymbol{X}} \boldsymbol{Y}\right)_{n} \in C^{\infty}(\sigma)
\end{aligned}
$$

is bilinear over the ring $C^{\infty}(\sigma)$.

Corollary. For every $\boldsymbol{x} \in \sigma$ formula (8.7) defines the bilinear mapping

$$
T_{\boldsymbol{x}}(\sigma) \times T_{\boldsymbol{x}}(\sigma) \ni(X, Y) \rightarrow S(X, Y) \in \mathbb{R} .
$$

Proposition 6. The mapping

$$
\begin{aligned}
& C^{\infty}(T(\sigma)) \times C^{\infty}(T(\sigma)) \ni(X, Y) \rightarrow \bar{D}_{\mathbf{X}} Y \\
& \quad=\left(D_{\mathbf{X}} \boldsymbol{Y}\right)_{t} \in C^{\infty}(T(\sigma))
\end{aligned}
$$

defines a covariant derivative on the manifold $\sigma$.

Proof. According to the axioms of covariant derivatives [12] we have to prove that for $f \in C^{\infty}(\sigma)$

$$
\overline{\boldsymbol{D}}_{f \mathbf{X}} \boldsymbol{Y}=f \overline{\boldsymbol{D}}_{\mathbf{X}} \boldsymbol{Y} ; \quad \overline{\boldsymbol{D}}_{\mathbf{X}}(f \boldsymbol{Y})=\boldsymbol{X}(f) \boldsymbol{Y}+f \overline{\boldsymbol{D}}_{\mathbf{X}} \boldsymbol{Y} .
$$

These properties follow directly from (8.8).

The covariant derivative $\overline{\boldsymbol{D}}$ on $\sigma$ defines a connection $\overline{\boldsymbol{\Gamma}}$ on $\sigma$. We call it the connection induced by the imbedding $i: \sigma \rightarrow M$. 
Definition. The mapping

$$
\begin{aligned}
& T_{\boldsymbol{x}}(\sigma) \times T_{\boldsymbol{x}}(\sigma) \ni(\boldsymbol{X}, \boldsymbol{Y}) \rightarrow \boldsymbol{K}(\boldsymbol{X}, \boldsymbol{Y}) \\
& \quad=-\boldsymbol{g}\left(\left(\boldsymbol{D}_{\mathbf{X}} \boldsymbol{n}\right)_{t}, \boldsymbol{Y}\right)=-\overline{\boldsymbol{g}}\left(\left(\boldsymbol{D}_{\mathbf{X}} \boldsymbol{n}\right)_{t}, \boldsymbol{Y}\right) \in \mathbb{R}
\end{aligned}
$$

is called the second fundamental form of the imbedding. The mapping

$$
T_{\boldsymbol{x}}(\sigma) \ni \boldsymbol{X} \rightarrow \boldsymbol{F}(\boldsymbol{X})=\left(\boldsymbol{D}_{\mathbf{X}} \boldsymbol{n}\right)_{n} \in \mathbb{R}
$$

is called the fundamental 1 -form of the imbedding.

\section{Proposition 7.}

$$
\begin{aligned}
& F(X)=\frac{1}{2}\left(D_{X} g\right)(n, n), \\
& S(X, Y)-K(X, Y)=-\left(D_{X} g\right)(Y, n) .
\end{aligned}
$$

\section{Proposition 8.}

$$
S(X, Y)-S(Y, X)=g(Q(X, Y), n),
$$

where $\boldsymbol{Q}=\left(Q_{\mu \nu}^{\lambda}\right)$ is the torsion tensor of $\boldsymbol{\Gamma}$,

$$
Q(X, Y)=D_{X} Y-D_{Y} X-[X, Y]
$$

cf. [12].

Corollary. For a symmetric connection $\boldsymbol{\Gamma}$ which preserves the scalar product i.e. $\boldsymbol{D} \boldsymbol{g}=0$ we have $\boldsymbol{F}=0, \boldsymbol{S}=\boldsymbol{K}$ and $\boldsymbol{K}$ is a symmetric 2 covariant tensor field on $\sigma$.

The torsion tensor $\overline{\boldsymbol{Q}}$ of the connection $\overline{\boldsymbol{\Gamma}}$ on $\sigma$ is given by

\section{Proposition 9.}

$$
\bar{Q}(X, Y)=(\boldsymbol{Q}(\boldsymbol{X}, \boldsymbol{Y}))_{t} .
$$

For the covariant derivative $\overline{\mathbf{D}}$ of the tensor $\overline{\boldsymbol{g}}$ on $\sigma$ we have

\section{Proposition 10.}

$$
\left(\bar{D}_{\mathbf{Z}} \bar{g}\right)(X, Y)=\left(D_{\mathbf{Z}} \boldsymbol{g}\right)(X, Y) ; \quad X, Y, Z \in T(\sigma) .
$$

Corollary. If $\boldsymbol{\Gamma}$ is the pseudoriemannian connection corresponding to the metric $\boldsymbol{g}$ then $\overline{\boldsymbol{\Gamma}}$ is the riemannian connection corresponding to $\overline{\boldsymbol{g}}$.

We express now the above formulas in local coordinate system $\left(x^{\lambda}\right)$ for which $\sigma=\left\{\boldsymbol{x} \in M: x^{0}=0\right\}$. We have $\boldsymbol{X}=\left(0, X^{k}\right), \boldsymbol{Y}=\left(0, Y^{k}\right)$ and

\section{Proposition 11.}

$$
\begin{gathered}
\boldsymbol{F}(\boldsymbol{X})=X^{k}\left(r_{k 0}^{0}-N^{s} r_{k s}^{0}\right) \\
\boldsymbol{K}(\boldsymbol{X}, \boldsymbol{Y})=-X^{k}\left(D_{k} n^{\mu}\right) g_{\mu s} Y^{s} \\
\boldsymbol{S}(\boldsymbol{X}, \boldsymbol{Y})=-N \Gamma_{k s}^{0} X^{k} Y^{s} \\
\left(\overline{\boldsymbol{D}}_{\boldsymbol{X}} \boldsymbol{Y}\right)^{s}=X^{k}\left(\partial_{k} Y^{s}+\bar{\Gamma}_{k r}^{s} Y^{r}\right)
\end{gathered}
$$


where

$$
\bar{\Gamma}=\left(\bar{\Gamma}_{k r}^{s}\right), \quad \bar{\Gamma}_{k r}^{s}=\Gamma_{k r}^{s}+N^{s} \Gamma_{k r}^{0} .
$$

Remark. We see from (8.20) and (A.2.8) that the components of the connection $\bar{\Gamma}$ on $\sigma$ are the spatial components of the connection $\Gamma$ taken in the normal coordinate system.

For the fundamental mappings we have in the normal coordinates $\boldsymbol{F}=\left(F_{k}\right)$, $\boldsymbol{K}=\left(K_{i j}\right), \boldsymbol{S}=\left(S_{i j}\right), \overline{\mathbf{D}}=\left(\bar{D}_{k}\right)$

$$
\begin{aligned}
F_{k} & =\bar{r}_{k 0}^{0}, \\
K_{i j} & =-\bar{\Gamma}_{i 0}^{s} g_{s j}, \\
S_{i j} & =-\bar{\Gamma}_{i j}^{0}, \\
\bar{D}_{k} Y^{s} & =\partial_{k} Y^{s}+\bar{\Gamma}_{k r}^{s} Y^{r} .
\end{aligned}
$$

\section{Geometric Interpretation of the Canonical Variables}

In Section 4 we have presented the geometric construction of canonical variables for the matter field $\varphi=\left(\varphi^{A}\right)$. We explain now the geometric meaning of the gravitational variables $\left(\Pi^{p q}, g_{p q}\right)$. We know that the variables $g_{p q}$ are components of a metric tensor on the submanifold $\sigma \subset M$. To elucidate the interpretation of $\Pi^{p q}$ we note that it follows from (2.8) and (5.3)

\section{Lemma 1.}

$$
\begin{aligned}
\Pi^{p q}= & \pi^{p q}+\frac{1}{2} \sqrt{-g}\left(r_{\tau \sigma}^{\tau} g^{\sigma 0}-r_{\alpha \beta}^{0} g^{\alpha \beta}\right) \bar{g}^{p q} \\
& +\frac{1}{2} \sqrt{-g}\left(\bar{g}^{a p} \bar{g}^{b q}+\bar{g}^{a q} \bar{g}^{b p}\right) r_{a b}^{0} .
\end{aligned}
$$

Using (9.1), (A.2.8), and (A.3.5) we have in the normal coordinate system

\section{Proposition 12.}

$$
\Pi^{p q}=\frac{1}{2} \sqrt{\bar{g}}\left(\bar{\Gamma}_{a b}^{0}\left(\bar{g}^{a p} \bar{g}^{b q}+\bar{g}^{a q} \bar{g}^{b p}\right)-\bar{g}^{p q}\left(\bar{\Gamma}_{a b}^{0} \bar{g}^{a b}+\bar{\Gamma}_{p 0}^{p}\right)\right) .
$$

If we compare $(9.2)$ with $\left(8.16^{\prime}\right)-\left(8.19^{\prime}\right)$ we have

\section{Theorem 2.}

$$
\Pi^{p q}=-\frac{1}{2} \sqrt{\bar{g}}\left(S_{a b}\left(\bar{g}^{a p} \bar{g}^{b q}+\bar{g}^{a q} \bar{g}^{b p}\right)-\bar{g}^{p q}\left(S_{a b} \bar{g}^{a b}+K_{a b} \bar{g}^{a b}\right)\right) .
$$

Formula (9.3) is a generalization of (5.2), in fact if $\Gamma_{\mu \nu}^{\lambda}=\left\{\begin{array}{c}\lambda \\ \mu \nu\end{array}\right\}$ then we have from Proposition $8 K_{a b}=S_{a b}, K_{a b}=K_{b a}$, and $\Pi^{p q}=\pi^{p q}$.

\section{Appendix 1}

Let $V$ be a manifold parametrized by symmetric quantities $\left(g_{\mu \nu}\right),\left(g_{\mu \nu}=g_{v \mu}\right)$. We can formally treat all variables $\left(g_{\mu \nu}\right)$ as independent. Therefore

$$
\frac{\partial g_{\alpha \beta}}{\partial g_{\mu \nu}}=\delta_{\alpha}^{\mu} \delta_{\beta}^{\nu} .
$$


For a function $F$ on $V$ (symmetric in $g_{\mu \nu}$ ) we have

$$
\partial F=\frac{\partial F}{\partial g_{\mu \nu}} d g_{\mu \nu}, \text { where } \frac{\partial F}{\partial g_{\mu \nu}}=\frac{\partial F}{\partial g_{v \mu}} .
$$

A vector $X$ tangent to $V$ can be written

$$
X=\delta g_{\mu \nu} \frac{\partial}{\partial g_{\mu \nu}}, \quad \delta g_{\mu \nu}=\delta g_{\nu \mu} .
$$

We have the natural pairing of $\frac{\partial}{\partial g_{\mu \nu}}$ and $d g_{\alpha \beta}$

$$
\left\langle\frac{\partial}{\partial g_{\mu \nu}} \mid d g_{\alpha \beta}\right\rangle=\delta_{\alpha}^{\mu} \delta_{\beta}^{v}
$$

In the papers [17-19] we took another convention, and treated only $g_{\mu \nu} \mu \leqq v$ as independent variables. In such a situation

$$
\begin{aligned}
\frac{\partial g_{\alpha \beta}}{\partial g_{\mu \nu}} & =\delta_{\alpha}^{\mu} \delta_{\beta}^{v}+\delta_{\alpha}^{v} \delta_{\beta}^{\mu}, \quad \mu \neq v ; \quad=\delta_{\alpha}^{\mu} \delta_{\beta}^{v}, \quad \mu=v \\
d F & =\sum_{\mu \leqq \nu} \frac{\partial F}{\partial g_{\mu \nu}} d g_{\mu \nu} ; \quad X=\sum_{\mu \leqq v} \delta g_{\mu \nu} \frac{\partial}{\partial g_{\mu \nu}} ; \\
\left\langle\frac{\partial}{\partial g_{\mu \nu}} \mid d g_{\alpha \beta}\right\rangle & =\delta_{\alpha}^{\mu} \delta_{\beta}^{v}+\delta_{\alpha}^{v} \delta_{\beta}^{\mu}, \quad \mu \neq v ; \quad=\delta_{\alpha}^{\mu} \delta_{\beta}^{v}, \quad \mu=v .
\end{aligned}
$$

\section{Appendix 2 (The Normal Coordinate System)}

Let $\left(x^{\lambda}\right)$ be local coordinates on $M$ and $\sigma=\left\{\boldsymbol{x} \in M: x^{0}=0\right\}$. Let $\boldsymbol{g}=\left(g_{\mu \nu}\right)$ be a pseudoriemannian metric on $M$ and $\sigma$ be space-like with respect to $g$. The normal unit vector to $\sigma \boldsymbol{n}=\left(1 / N,-N^{k} / N\right)$. A normal local coordinate system $\left(x^{\bar{\lambda}}\right)$ in a neighbourhood of $\sigma$ in $M$ is given by

$$
x^{\overline{0}}=N\left(x^{\lambda}\right) x^{0}, \quad x^{\bar{k}}=x^{k}+x^{0} N^{k}\left(x^{\lambda}\right)
$$

and conversely

$$
x^{0}=x^{\overline{0}} / N\left(x^{\bar{\lambda}}\right), \quad x^{k}=x^{\bar{k}}-x^{\overline{0}} N^{k}\left(x^{\bar{\lambda}}\right) / N\left(x^{\bar{\lambda}}\right) .
$$

We have

$$
\begin{array}{lll}
\frac{\partial x^{\overline{0}}}{\partial x^{0}}=N, & \frac{\partial x^{\bar{k}}}{\partial x^{0}}=N^{k}, & \frac{\partial x^{\bar{\mu}}}{\partial x^{s}}=\delta_{s}^{\mu} \\
\frac{\partial x^{0}}{\partial x^{\overline{0}}}=1 / N, & \frac{\partial x^{k}}{\partial x^{\overline{0}}}=-N^{k} / N, & \frac{\partial x^{\mu}}{\partial x^{\bar{s}}}=\delta_{s}^{\mu} .
\end{array}
$$

In (A.2.2) partial derivatives are taken at a point $\boldsymbol{x} \in \sigma$ i.e. $x^{0}=x^{\overline{0}}=0$. 
According to the Definition (4.1) for a scalar $\varphi$ on $M \bar{\varphi}=\varphi \mid \sigma$ (the restriction of $\varphi$ to $\sigma$ ) for a vector $v^{\lambda}$ we have
a scalar $v^{\overline{0}}=N v^{0}$,
a vector (tangent to $\sigma$ ) $v^{\bar{k}}=v^{k}+v^{0} N^{k}$.

For a covector $p_{\lambda}$ we have

a scalar $\quad \bar{p}_{0}=(1 / N) p_{0}-\left(N^{s} / N\right) p_{s}$

a covector $\bar{p}_{k}=p_{k}$.

For a tensor $b_{v}^{\mu}$ we have

$$
\begin{array}{ll}
\text { scalar } \quad \bar{b}_{0}^{0} & =b_{0}^{0}-N^{s} b_{s}^{0} \\
\text { covector } \bar{b}_{s}^{0} & =N b_{s}^{0} \\
\text { vector } \quad \bar{b}_{0}^{k} & =b_{0}^{k}(1 / N)+b_{0}^{0}\left(N^{k} / N\right)-b_{s}^{0}\left(N^{k} N^{s} / N\right)-b_{s}^{k}\left(N^{s} / N\right) \\
\text { tensor } \quad \bar{b}_{s}^{k} & =b_{s}^{k}+b_{s}^{0} N^{k} .
\end{array}
$$

For a tensor $z_{\lambda}^{\mu \nu}$ we have

$$
\text { tensor } \begin{aligned}
\bar{z}_{0}^{p q}= & z_{0}^{p q}(1 / N)+z_{0}^{00}\left(N^{p} N^{q}\right. \\
& +z_{0}^{0 q}\left(N^{p} / N\right)-z_{s}^{00}\left(N^{p} N^{q} N^{s} / N\right)-z_{s}^{p \cup}\left(N^{q} N^{s} / N\right) \\
& -z_{s}^{0 q}\left(N^{p} N^{s} / N\right)-z_{s}^{p q}\left(N^{s} / N\right)
\end{aligned}
$$

tensor $\bar{z}_{s}^{p 0}=z_{s}^{p 0} N+z_{s}^{00} N N^{p} \quad\left(\mathrm{cf} .\left(5.3^{\prime}\right)\right)$ and so on.

For a tensor $r_{\mu \nu}^{\lambda}$ we have

$$
\begin{array}{ll}
\text { covector } & \bar{r}_{p 0}^{0}=r_{p 0}^{0}-r_{p q}^{0} N^{q} \\
\text { tensor } & \bar{r}_{p q}^{0}=N r_{p q}^{0} \quad \mathrm{cf} \text { (8.16) and so on. }
\end{array}
$$

For coefficients of a connection $\Gamma$ on $M$ we have

$$
\bar{\Gamma}_{\mu \nu}^{\lambda}=\Gamma_{\bar{\mu} \bar{\nu}}^{\bar{\lambda}}=\frac{\partial x^{\bar{\lambda}}}{\partial x^{\lambda}} \frac{\partial x^{\mu}}{\partial x^{\bar{\mu}}} \frac{\partial x^{\nu}}{\partial x^{\bar{\nu}}} \Gamma_{\mu \nu}^{\lambda}+\frac{\partial^{2} x^{\sigma}}{\partial x^{\bar{\mu}} \partial x^{\bar{\nu}}} \frac{\partial x^{\bar{\lambda}}}{\partial x^{\sigma}} .
$$

In particular case of the riemannian connection $\gamma_{\mu \nu}^{\lambda}=\left\{\begin{array}{c}\lambda \\ \mu \nu\end{array}\right\}$ we have

$$
\begin{aligned}
& \bar{\gamma}_{0 s}^{0}=0, \quad \bar{\gamma}_{p q}^{0}=N \gamma_{p q}^{0}, \quad \bar{\gamma}_{q 0}^{p}=N \bar{g}^{p u} \gamma_{u q}^{0} \\
& \bar{\gamma}_{p q}^{s}=N^{s} \gamma_{p q}^{0}+\gamma_{p q}^{s} \quad \text { and so on. }
\end{aligned}
$$

Remark. The above definition of $\bar{\gamma}_{p q}^{s}$ coincides with that given in Appendix 3 . We have also

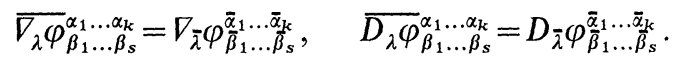

The covariant derivative of the quantity $\bar{\varphi}^{A}$ is

$$
\begin{aligned}
\bar{\nabla}_{r} \bar{\varphi}_{\beta_{1} \ldots \beta_{s}}^{\alpha_{1} \ldots \alpha_{k}}= & \partial_{r} \bar{\varphi}_{\beta_{\beta_{1} \ldots \beta_{s}}^{\alpha_{1} \ldots \alpha_{k}}} \\
& +\delta_{z}^{\alpha_{1}} \bar{\gamma}_{r u}^{z} \bar{\varphi}_{\beta_{1} \ldots \beta_{s}}^{u \alpha_{2} \ldots \alpha_{k}}+\ldots+\delta_{z}^{\alpha_{k}} \bar{\gamma}_{r u}^{z} \bar{\varphi}_{\beta_{1} \ldots \beta_{s}}^{\alpha_{1} \ldots \alpha_{k}-1 u} \\
& +-\delta_{\beta_{1}}^{q} \bar{\gamma}_{r q}^{p} \bar{\varphi}_{p \beta_{2} \ldots \beta_{s}}^{\alpha_{1} \ldots \alpha_{k}}-\ldots-\delta_{\beta_{s}}^{q} \bar{\gamma}_{r q}^{p} \bar{\varphi}_{\beta_{1} \ldots \beta_{s}-1}^{\alpha_{1} \ldots \alpha_{k}} .
\end{aligned}
$$




\section{Appendix 3}

On the submanifold $\sigma=\left\{\boldsymbol{x} \in M: x^{0}=0\right\}$ we have

$$
\begin{aligned}
N= & \left(-g^{00}\right)^{-1 / 2}, \quad N_{k}=g_{0 k}, \quad N^{s}=\bar{g}^{s k} N_{k} \\
g^{p q}= & \bar{g}^{p q}-N^{p} N^{q} / N^{2}, \quad g_{00}=-N^{2}+N^{s} N_{s}, \quad g^{0 p}=N^{p} / N^{2} \\
g= & \operatorname{det} g_{\mu \nu}, \quad \bar{g}=\operatorname{det} g_{i j}, \quad \sqrt{-g}=N \sqrt{\bar{g}}, \\
\gamma_{\mu \nu}^{\lambda}= & \left\{\begin{array}{c}
\lambda \\
\mu \nu
\end{array}\right\}, \quad \bar{\gamma}_{p q}^{k}=\left\{\begin{array}{c}
k \\
p q
\end{array}\right\} \quad[\mathrm{cf} .(\mathrm{A} .2 .9)], \\
\gamma_{p q}^{k}= & \bar{\gamma}_{p q}^{k}-N^{k} \gamma_{p q}^{0} ; \quad \gamma_{p 0}^{0}=N^{s} \gamma_{s p}^{0}+(1 / N) \partial_{p} N \\
\gamma_{p 0}^{k}= & N^{2} \bar{g}^{k u} \gamma_{p u}^{0}+\bar{\nabla}_{p} N^{k}-N^{k} N^{u} \gamma_{p u}^{0}-N^{k} / N \partial_{p} N \\
\gamma_{00}^{0}= & \left(N^{a} / N\right) \partial_{a} N+(1 / N) \partial_{0} N+N^{a} N^{b} \gamma_{a b}^{0} \\
\gamma_{00}^{s}= & \partial_{0} N^{s}+N\left(\bar{g}^{s a}-N^{s} N^{a} / N^{2}\right) \partial_{a} N+N^{a} \bar{\nabla}_{a} N^{s} \\
& +2 N^{2} N^{p} \bar{g}^{s q} \gamma_{p q}^{0}-N^{s} / N \partial_{0} N-N^{s} N^{a} N^{b} \gamma_{a b}^{0}, \\
K_{i j}= & -N \gamma_{i j}^{0} ; \quad \pi^{i j}=\sqrt{-g}\left(\gamma_{p q}^{0}-g_{p q} \gamma_{a b}^{0} \bar{g}^{a b}\right) \bar{g}^{p i} \bar{g}^{q j} \\
\gamma_{p q}^{0}= & (-g)^{-1 / 2}\left(\pi_{p q}-\frac{1}{2} g_{p q} \operatorname{tr} \pi\right), \quad \operatorname{tr} \pi=g_{p q} \pi^{p q}, \\
\partial_{\lambda} g_{\mu \nu}= & g_{\mu \alpha} \gamma_{v \lambda}^{\alpha}+g_{v \alpha} \gamma_{\mu \lambda}^{\alpha} \\
\partial_{0} g_{i j}= & \bar{\nabla}_{i} N_{j}+\bar{\nabla}_{j} N_{i}+2 N^{2} \gamma_{i j}^{0} .
\end{aligned}
$$

Remark. $\bar{\nabla}_{r}$ denotes the covariant derivative with respect to the connection $\bar{\gamma}_{p q}^{k}$ on $\sigma$.

Acknowledgements. The author would like to express his thanks to Professor A.Trautman, Professor F.W.Hehl, Dr. W.Kopczyński for several discussion and critical remarks, to Dr. J.Kijowski for his interest in this work.

The main results of the paper were obtained during the author's visit at the University of Trieste and the International Centre for Theoretical Physics 1976/77. The author is very indebted to these institutions for their hospitality and to Consiglio Nazionale delle Ricerche for the financial support. Special thanks are due to Professor M.Dolcher for his help and cordiality in Trieste.

\section{References}

1. Adler, R., Bazin, R., Schiffer, M. : Introduction to general relativity. New York: McGraw Hill 1965

2. Arnowitt, R., Deser,S., Misner,C.W.: The dynamics of general relativity. In: Gravitation-an introduction to current research (ed. L. Witten). New York: John Wiley 1962

3. Fischer, A., Marsden,J.: Topics in the dynamics of general relativity. In: Proceedings of the Summer School of the Italian Physical Society (ed. J. Ehlers). Varenna 1976

4. García,P.L.: The Poincaré-Cartan invariant in the calculus of variations. In: Symposia mathematica 14, 219-246 (1974)

5. García,P.L.: Reducibility of the symplectic structure of classical fields with gauge symmetry. In: Differential geometrical methods in mathematical physics (ed. K. Bleuler, A. Reetz). Lecture notes in mathematics, Vol. 570. Berlin-Heidelberg-New York: Springer 1977

6. Goldschmidt,H., Sternberg, S. The Hamilton-Cartan formalism in the calculus of variations. Ann. Inst. Fourier 23, 203-267 (1973)

7. Hehl,F.W., Heyde,P., Kerlick,G.D., Nester,J.M.: General relativity with spin and torsion: foundation and prospects. Rev. Mod. Phys. 48, 393-416 (1976) 
8. Hehl,F.W., Kerlick, G.D., Heyde,P.: On hypermomentum in general relativity: Z. Naturforsch. 31a, 111-114, 524-527, 823-827 (1976)

9. Kijowski,J.: A finite dimensional formalism in the classical field theory. Commun. math. Phys. 30, 99-128 (1973)

10. Kijowski,J., Szczyrba,W.: A canonical structure for classical field theories. Commun. math. Phys. 46, 183-206 (1976)

11. Kijowski,J., Tulczyjew,W.M.: Potentiality, reciprocity and field theory. Lectures notes in physics (to appear)

12. Kobayashi,S., Nomizu,K.: Foundations of differential geometry, Vols. 1 and 2. New York: Interscience Publ. 1963, 1969

13. Kopczyński,W.: The Palatini principle with constraints. Bull. Acad. Polon. Sci. 23, 468-473 (1975)

14. Marsden,J., Fischer, A. : General relativity as a Hamiltonian system. In : Symposia mathematica 14, $193-205(1974)$

15. Misner,C.W., Thorne,K.S., Wheeler,J.A.: Gravitation. San Francisco: W.H. Freeman 1973

16. Szczyrba,W.: Lagrangian formalism in the classical field theory. Ann. Pol. Math. 32, 145-185 (1976)

17. Szczyrba,W.: A symplectic structure on the set of Einstein metrics. Commun. math. Phys. 51, $163-182(1976)$

18. Szczyrba,W.: On geometric structure of the set of solutions of Einstein equations. Dissertationes Mathematicae 150, 1-83 (1977)

19. Szczyrba, W.: A symplectic structure for the Einstein-Maxwell field. Rep. Math. Phys. 12, 169-191 (1977)

20. Szczyrba,W.: The field equations and contracted Bianchi identities in the generalized EinsteinCartan theory. Letters Math. Phys. (to appear)

21. Trautman, A.: On the structure of the Einstein-Cartan equations. In: Symposia mathematica 12, 139-162 (1973)

22. Trautman, A.: Recent advances in the Einstein-Cartan theory of gravity. Ann. New York Acad. Sci. 262, 241-245 (1975)

23. Wheeler,J.A.: Geometrodynamics and the issue of the final state. In: Relativity, groups and topology (ed. B. De Witt, C. De Witt). New York: Gordon Breach 1964

Communicated by R. Geroch

Received January 3, 1978 